\title{
La comunicación y los desafíos del mundo globalizado: categorías de análisis para la construcción de nuevas utopías*
}

\author{
Ludmila Maia Strycek $^{*}$
}

\section{Resumen}

El primer problema que podemos formular es acerca de la relación real, que existe o debería existir entre los medios masivos y los procesos sociales. Esto obviamente corta toda posibilidad de legitimar los procesos culturales "nacidos" de los nuevos escenarios. Por otro lado, es importante hacer una diferencia sustancial para abordar la problemática, siguiendo a Vizer (2003), de las construcciones sociales de sentido: información no es lo mismo que conocimiento.

Desde el punto de vista de un mundo globalizado, no queda espacio más que para la comprensión de los escenarios de poder público y político y la lucha por la "dominación" del escenario de construcción comunicacional. El Estado y el sector privado están en una permanente lucha. Las palabras clave para comprender la construcción de mundo a través de los medios, teniendo en cuenta los procesos sociales y culturales y el papel de los poderes hegemónicos, son: PRESENTACIÓN Y REPRESENTACIÓN.

Uno de los principales inconvenientes de la investigación en comunicación radica en la dificultad de conceptualizar a la comunicación en términos que no solo posibiliten una mirada crítica sobre los fenómenos que estudia, sino que posicionen a la disciplina en un campo que le permita tener una mirada

\footnotetext{
* Artículo recibido el 15 de marzo de 2013. Aceptado el 30 de julio de 2013.

* Diseñadora Gráfica por la Universidad Nacional del Nordeste.

Contacto: puntograficomax@gmail.com
} 
holística del problema, permitiéndole diagnosticar situaciones desde el aporte de otras disciplinas y a la vez, tener la capacidad de orientar las investigaciones en los caminos correctos, esto es, con la mirada puesta en el futuro, construyendo nuevas utopías.

\section{Palabras clave}

Comunicación - Globalización - Sociedad de consumo Democracia - Construcciones culturales.

\section{Abstract}

The first problem we have to formulate is about the real relationship that exists or should exist between mass media y social processes. Obviously, this eliminate every possibility of legitimize cultural processes that were "born" from the new stages. On the other hand, it is important to make a substantial difference to approach to the problematic of social sense construction: information is not the same that knowledge.

From the point of view of a Globalize world, there is just space for understanding of public and political stages and the battle for "denomination" of communicational construction stage. The Government and private sector are constantly struggling. The key words to understand the construction of a world by mass media, considering cultural and social processes and the rol of hegemonic powers, are: PRESENTATION and REPRESENTATION. One of the principal drawbacks of the communication research, is in the hard work of conceptualize communication in terms that, not only makes possible a critical view on studying phenomena, but it puts the discipline in a field that can be able to have an holistic view of the problem, enabling it to diagnose some situations from the contribution of other disciplines and at the same time, to be able to guide researches to the right ways, this mean, looking to the future, building news utopias.

\section{Keywords}

Communication - globalization - consumer society - democracy - cultural constructions. 


\section{Introducción}

La problemática de la investigación en temas de comunicación en América Latina propone varios desafíos. Por un lado, se hace difícil delimitar en estos días cuál es el objeto de estudio concreto y definitivo de la comunicación, y posiblemente, lleguemos a la conclusión de que no existe tal. Por otro lado, se encuentran todos los conceptos ligados a la definición de una epistemología de las ciencias sociales, que aun no ha podido, desde mi punto de vista, resolver viejas preguntas filosóficas, ligadas primordialmente a la definición de "realidad", posicionando no solo al investigador, sino también al epistemólogo en una disyuntiva permanente: la de tener que optar por definir cuál es el nivel de intervención/interpretación/construcción de la realidad que tiene la comunicación, o la de avanzar en las problemáticas concretas que lo apuran desde otras perspectivas y con otros requerimientos, que a pesar de estar íntimamente ligados, reclaman otras miradas más profundas y superadoras. Por otra parte, se ha desdibujado la verdadera mecánica y motivación de la ciencia (como institución), en temas de investigación de las ciencias sociales, sobre todo a partir de la hegemonía del positivismo. En los últimos años, sin embargo, se ha hecho el esfuerzo por conformar una visión científica más amplia, incluyendo los factores sociológicos de base. Esto también implica una nueva serie de problemáticas ligadas a la "ligereza" con la que se pueden llegar a abordar las investigaciones. A este respecto, dice Follari:

La ciencia es ideológica, entonces, y alberga inevitablemente valoración. Sin embargo, esto no puede entenderse como una especie de coartada para la flojedad epistemológica, la pérdida del rigor metodológico o la apelación a una especie de ausencia de restricciones y exigencias. Que la conquista de la objetividad sea siempre parcial y se haga desde un punto de vista teórico determinado, no podría en ningún caso confundirse con una especie de apertura a la simple descripción, o de rendición a la libre opinión y la carencia de mediaciones conceptuales y empíricas precisas. (Follari 2002, p. 2).

Raúl Fuentes Navarro ${ }^{1}$, ha analizado los temas más relevantes tratados en las investigaciones en ciencias sociales en América Latina. Afirma que se desarrollan en tres campos articulados:

1) El de la historia del campo (sugiere relecturas y reescrituras que orienten a la renovación de las utopías fundantes).

\footnotetext{
${ }^{1}$ FUENTES NAVARRO, Raúl. La investigación de la comunicación en América Latina: condiciones y perspectivas para el SXXI, en Diálogos, Felafacs, Lima.
} 
2) El plano propiamente científico (pertinencia de una metodología comunicacional como eje para articular la teoría y la práctica de la investigación en búsqueda de una mayor consistencia epistemológica y una mayor pertinencia ética).

3) El plano de la construcción comunitaria del futuro.

Afirma que "En América Latina, más que nada, parece haberse perdido la profundidad ideológica, el poder de las creencias que orienten las búsquedas del sentido de la comunicación". Nancy Díaz Larrañaga y Paula Inés Porta ${ }^{2}$ afirman en este sentido que a la vez que se modifica lo social, es decir, el objeto de las ciencias sociales, lo hace correlativa y simultáneamente la subjetividad de quienes lo estudian.

Para repensar las ciencias sociales hay que abandonar dos posturas clásicas: la conservadora, que "consiste en tomar los clásicos como fundadores de un saber acabado", y la opuesta, que propone que todo lo hecho hasta hora es incompatible con lo que ya se ha pensado.

\section{Materiales y métodos}

Este artículo consiste en una revisión bibliográfica tanto de autores latinoamericanos, como europeos, que se encuentran analizando y cuestionando el problema de la comunicación desde una perspectiva epistemológica.

Se compilaron las fuentes que abordaban la problemática de la comunicación y se analizaron desde un punto de vista crítico, se aislaron las ideas principales para luego ser contrastadas teóricamente, tratando de vincularlas con la realidad latinoamericana, a los fines de generar categorías de análisis de la realidad científica ligada a la comunicación.

Para ello, se aislaron dos grandes ejes de discusión: "La intersección entre Comunicación y Modernidad" y la relación entre "Los medios masivos y los procesos sociales". Estos ejes agrupan las principales ideas que rondan en torno a la mirada epistémica de la investigación en ciencias sociales, y fundamentan la discusión sobre los paradigmas existentes y las utopías que se deben construir a futuro.

\section{Discusión de resultados}

I. La intersección entre Comunicación y Modernidad

El principal problema de la comunicación es definir qué es comunicación dentro de los contextos actuales. Abordar una definición de este término en relación con la dualidad Comunicación/Modernidad implica sostener un debate teórico muy amplio y que en gran parte, como lo indica Wolton (1999), nos ha sido negado, y nos lo hemos negado (sobre todo los comunicadores, siendo esta parte de nuestra responsabilidad).

\footnotetext{
2 DÍAZ LARRAÑAGA, Nancy; PORTA, Paula Inés. (2011) "Clases del 1 al 11: EPISTEMOLOGÍA Y TRAYECTOS TEÓRICOS COMUNICACIONALES". UNQ. (Inédito)
} 
En primer lugar, es más que obvio que el modelo que hay que considerar poner en crisis (para acordar erradicarlo de una vez por todas) es el de emisor-mensaje-receptor, porque ya no basta (desde hace ya casi un siglo) ni para poder describir, ni analizar, ni discutir las dinámicas de las comunicaciones actuales. Esta es una postura ampliamente positivista, responde a la dinámica estricta del modelo moderno, pero que ha demostrado haber sido insuficiente, y a la vez errónea, para la observación de fenómenos sociales.

Si concebimos a la comunicación no como un fenómeno aislado sino como un proceso de intercambio y de re-construcción cultural y social, podemos estar en condiciones de posicionar a la comunicación en el lugar esencial de la evolución constante de la sociedad moderna. Ahora bien, cabe preguntarse en ese caso: qué estuvo primero, la comunicación como un proceso necesario al entendimiento y al intercambio (por lo tanto, a la supervivencia) o el requerimiento del medio, considerado como una tecnología de la información (siendo esta, dentro del pensamiento de Mac Luhan, "una extensión del cuerpo" de configuración esencialmente artificial).

Si hacemos "arqueología" de la comunicación, podemos rastrear instrumentos tecnológicos utilizados para fines comunicativos hasta los confines de la historia.

En este punto, me parece oportuno plantear la primera afirmación: se considera COMUNICACIÓN a todo proceso INTENSIONAL, de construcción y mediatización de mensajes dentro de un contexto social y cultural determinado

Es importante aclarar que la cuestión de la intencionalidad nos puede ayudar a definir un modelo de comunicación acorde con la modernidad, en donde cada "emisión" de mensajes espera una respuesta real, rápida y eficiente dentro del mismo referente de la comunicación; no es posible afirmar que "es imposible no comunicar", sobre todo si entendemos a la comunicación como un "espacio" multidimensional y no como una configuración lineal.

El referente de la comunicación/modernidad bien podría situarse en la tríada sociedad de consumo + democracia de masas + medios masivos, pero sobre estos temas articulados no se ha dicho mucho; de hecho, se han convertido en una verdad innegable. Wolton ${ }^{3}$ afirma que

La paradoja de la comunicación es la siguiente: si la historia de la comunicación es evidentemente muy larga, tan larga como la del hombre, la de las tecnologías del mismo nombre es, por el contrario, extraordinariamente reciente. $Y$ los hombres todavía no se han habituado a unos sistemas de comunicación que cambian considerablemente su percepción del mundo, su modo de vida y de trabajo, y ya deben

\footnotetext{
3 WOLTON, Dominique (1999) "La comunicación en el centro de la modernidad. Un debate teórico fundamental". En Internet, ¿y después?: una teoría crítica de los nuevos medios de comunicación. Barcelona: Gedisa. pp. 35-67.
} 
prepararse para la etapa siguiente, en la que todo irá aún más rápido. $Y$, entonces, ¿por qué hay tan pocas discusiones y controversias sobre las tecnologías de comunicación? (Wolton 1999, p. 2).

Hay que comprender también que hoy en día el nivel de participación en la construcción de mensajes no es simétrico ni igualitario, ni tiene los mismos niveles de valor y jerarquía, como los pretende tener por ejemplo el sufragio; por lo tanto, no se puede hablar de una "democracia comunicacional" ni de "democracia de masas", en estos términos.

Para explicar esto, se puede tomar el concepto de "formas simbólicas" que propone Thompson (1998):

La comunicación de masas por lo general implica la producción de bienes para el consumo de formas simbólicas en el sentido de que estos objetos producidos por las instituciones mediáticas son formas simbólicas sujetas, de una u otra manera, al proceso de valoración económica. Los modos de valorar las formas simbólicas varían enormemente, dependiendo de los medios técnicos y de las estructuras institucionales dentro de las cuales se despliegan. (Thompson, 1998, p. 17)

Esta otra perspectiva intenta agregarle a la ecuación una visión que ayuda a la comprensión del sistema de construcción e interpretación de la información. Los mecanismos del "mercado" ponen en escena mensajes que requieren un esfuerzo por parte del espectador para comprenderlos, poniendo en funcionamiento, desde un punto de vista hermenéutico, procesos activos, creativos y preconceptos. Una vez más, este fenómeno apoya la idea de que existe en este modelo la innegable categoría de la intencionalidad, regulando y marcando el ritmo, si se quiere, del circuito comunicacional.

Por un lado, los medios masivos generan mensajes estratégicos, colocándolos en los "lugares precisos" en donde un grupo bien delimitado de receptores los puedan percibir y decodificar. Siguiendo a Thompson (1998) se puede decir que

muchas de las asunciones y expectativas que un individuo emplea en el proceso de interpretación poseen un amplio carácter social e histórico. Se trata de las asunciones $y$ expectativas de un grupo de individuos que comparten ampliamente orígenes sociales y trayectorias parecidas. Constituyen un tipo de conocimiento implícito que los individuos adquieren a través de un proceso gradual de inculcación, y que les ofrece una estructura para la interpretación y asimilación de lo nuevo.

Es decir que la "estrategia" implica también un reconocimiento del contexto social en el cual funciona el sistema de comunicación. 
Esto lleva a otra de de las preguntas fundamentales: “¿Por dónde pasa el recorte de la realidad que un investigador o una comunidad de investigadores define como legitimidad de un campo o un objeto de estudio?" (Vizer 2003, p.98)

Debemos revisar si realmente existe una definición de comunicación, ya que hasta ahora, a partir de la multiplicidad de posturas y de la complejidad de los fenómenos sociales y de sus dinámicas, no se ha podido abordar un concepto claro de ella.

Esto encierra varias cuestiones:

1. La construcción de sentido y la producción de significados: la realidad se articula entre los individuos, la cultura y la sociedad. Los contextos sociales y las acciones son creencias, significados, sentidos y certezas.

2. El concepto de acción social e interacción social (Max Weber), que aportan mayores datos para el entendimiento de los fenómenos que rodean no solo la comprensión sino la construcción misma de la realidad, como un fenómeno "socialmente" articulado (valga la redundancia). Y el aporte de Durkheim con sus teorías de la solidaridad mecánica y orgánica también puede echar luz acerca de la evolución del fenómeno de la comunicación.

3. La inclusión de las tecnologías de la información, como parte fundamental de la comprensión de dichos procesos como un fenómeno evolutivo: existe la posibilidad de "rastrear" los fenómenos de la mediatización de la información hasta los "confines" de la civilización. En este aspecto (Vizer et ál., 2003) aporta un concepto más profundo: el de determinar qué procesos institucionales se llevan a cabo en la construcción del sentido, en cuyo caso, se hace necesario agregar a nuestro problema la necesidad de saber qué instituciones tienen el poder de mediatizar la información, cómo lo hacen y con qué fines.

Paralelamente a esto está el problema de cómo se aborda, quién lo hace y con qué herramientas se cuenta para la explicación de los fenómenos: el punto de partida, la posición del científico para estudiar los fenómenos define la aproximación a la construcción de la realidad. El concepto mismo de realidad "como cosa en sí" o como "constructo fenomenológicamente aceptado" determina el conocimiento que se da acerca de la funciones de la comunicación y de su participación como instrumento para la generación de significados y de sentido socialmente compartido.

La postura científica también está en sintonía con los intereses políticos y económicos de un contexto social determinado. Así, no es casual que para los fines de capitalismo sea funcional la postura positivista, que solo se puede ocupar (por sus limitaciones, no solo instrumentales sino también ideológicas) de referirse o describir la INFORMACIÓN, en tanto que no hace mayores aportes acerca de la construcción, divulgación y "administración" del conocimiento.

Por un lado están las posturas de abordaje, y por el otro la representación de la realidad.

El cuarteto a tener en cuenta es: ciencia-poder-tecnología-economía. 
Los significados dependen del contexto. El sentido es una construcción social.

II. Vincular los medios masivos con los procesos sociales

El primer problema que podemos formular es acerca de la relación real que existe o debería existir entre los medios masivos y los procesos sociales. Estos últimos, en lugar de ser estudiados desde la óptica de la compresión, se limitan a estructurar críticas desde las diferentes perspectivas. Aquí se hacen oportunas las definiciones de estrategia y táctica propuestas por Michel De Certeau (1996): mientras que las diferentes disciplinas buscan comprender las situaciones de poder que se desarrollan y se implementan dentro de las instituciones y sectores dominantes hacia la ciudadanía, esta otra parece "resolver" sus problemáticas y construir sentido dentro de un escenario que le es ajeno, pero las construcciones que se desarrollan son genuinas, son absolutamente originales y propias; es por eso que resulta difícil la medición (histérica, obsesiva) de las ciencias sociales a los diferentes sectores, buscando reducir a la dualidad (oferta-demanda; social-antisocial; mediatizado-no mediatizado, estadosector privado etc.), siendo que la verdadera búsqueda está en la "relación" de los medios con los procesos sociales como una buena manera de legitimación de estos últimos. De esta forma es posible que se generen perspectivas más optimistas, ya que el punto de partida es válido.

Otro de los conceptos que se puede recuperar es aquel que postula, como lo indica Castoriadis (2006), que "la sociedad llega más allá del límite de lo instrumental. Evidentemente no hay ninguna sociedad que carezca de cultura; ninguna sociedad se ve reducida a lo funcional y a lo instrumental" (Castoriadis. 2006, p. 6). Al respecto, se puede aislar este concepto tomando en cuenta no solo la forma en la que se está haciendo investigación en relación con la comunicación, sino también con los valores que se aíslan como instalados por el "poder hegemónico" (el de la producción de objetos, en los que prima su función), siendo que de forma natural y necesaria existe también una producción cultural, que si no es étnica o histórica, no tiene posibilidades de validarse dentro de sus nuevas configuraciones. Esto obviamente corta toda posibilidad de legitimar los procesos culturales "nacidos" de los nuevos escenarios. Hay aquí una paradoja:

a) Los poderes institucionalizados generan estrategias de comunicación y validación culturales.

b) La ciudadanía responde generando y produciendo elementos culturales (formas de acción e interacción, comunicación, intercambio).

c) Al no haber una forma literal de entendimiento y traducción, las producciones sociales son aisladas de las nuevas estrategias mediáticas, abriendo cada vez más la brecha entre los escenarios y sus actores. 
Por otro lado, es importante hacer una diferencia sustancial para abordar la problemática de, (Vizer et ál., 2003), las construcciones sociales de sentido: información no es lo mismo que conocimiento.

La información no produce sentido ni aún significados, produce una materia prima, signos, textos. Solo la interpretación procesa la información con el fin de construir posibles significaciones, valores y sentidos. En otras palabras, las ciencias sociales cumplen la función de "metalenguajes" que tienen como objetivo explorar la complejidad "holística" de los procesos de construcción de la vida social de los hombres en tanto prácticas, acciones, representaciones y expresiones de sentido. (Vizer 2003, pp. 58-59)

El conocimiento, por otra parte, está relacionado con contextos de emisión administrados por grupos de poder. Esto no es tan extraño, se ajusta perfectamente a la teoría sociológica. La desconfianza de la que habla Wolton (1999) se liga fundamentalmente a dos cuestiones: la primera acerca de la importancia, eficiencia (en generar cambios o impactar sobre la opinión pública) y la legitimidad de la información; la segunda, acerca de la veracidad, contrastación, postura ideológica, capacidad de difusión y credibilidad del conocimiento.

Cabe recordar aquella vieja anécdota de Orson Wells transmitiendo por radio la invasión alienígena en 1938: inmediatamente la sociedad le atribuyó valores que se ligaron a la confiabilidad que tenía la radio en esos tiempos, como legítimo medio de comunicación. El experimento demostró, entre otras cosas, que la gente está dispuesta a creer cualquier tipo de información, por más irracional que parezca, si el contexto de emisión es verosímil.

La segunda palabra clave (siendo la primera "intención") es "edición": ya sea espontánea -ligada a los aspectos culturales y sociales-, como intencional. Los mensajes pueden torcer y reformular posturas, conceptos, prácticas, lo que los hace más poderosos es el nivel de credibilidad que tengan, que sustancialmente está ligado a los medios.

Los medios ayudan a la difusión (cada vez más rápida, más frenética) de información. La eficiencia de la comunicación, por lo tanto, está referida a los siguientes condicionantes:

- la credibilidad;

- la eficiencia en la codificación;

- la difusión y

- la accesibilidad a la tecnología.

Otros aspectos para tener en cuenta: "El modelo de comunicación de la educación está cien años más atrás que el modelo de comunicación social" (Martin Barbero, 2010). 
Aquí es válida la recuperación de lo que indica el autor, que contribuye a la explicación de la instalación de un tipo de pesimismo, ligado a la incomprensión de las dinámicas comunicacionales a nivel social y de su relación con los medios. Es importante la distinción (o aclaración) que hace el autor en cuanto a los instrumentos de los medios, afirmando que no se debe comprender "a través de ellos", sino cómo funcionan los procesos "con" ("haciendo uso de") ellos. No es una problemática de los medios o de la tecnología, sino una problemática de las ciencias sociales, que no han encontrado el modelo de análisis, de comprensión, y de la educación, que se sigue manteniendo ajena a la discusión. La educación ya no es más capaz de responder a las necesidades del mercado, pero no desde el punto de vista del consumo de bienes, sino desde el punto de vista del ¿consumo? de medios y de los procesos sociales ligados a esto (por lo tanto, de interacción y de producción e intercambio).

Esto también tiene que ver con el ejercicio de los derechos y de la concepción democrática de la sociedad:

El individuo democrático no puede existir si no está lúcido, en primer lugar, con respecto a sí mismo. Esto no significa que sea necesario psicoanalizar a todo el mundo. Pero habrá que llevar a cabo, quizás, una reforma radical de la educación, que consistiría, entre otras cosas, en tener en cuenta en grado mucho mayor la cuestión de la autonomía de los alumnos, incluyendo sus dimensiones psicoanalíticas, cosas que hoy no ocurre. (Michel De Certeau, 1997)

\section{Globalización}

El problema de la globalización siempre ha sido estudiado desde las perspectivas del impacto: económico (ligado a los cambios y a las relaciones de consumo), cultural (desde la perspectiva de la interculturalidad vista como una especie de "invasión" más que de re-formulación de dinámicas), social e interinstitucional.

Desde esta visión de la globalización, no queda espacio más que para la comprensión de los escenarios de poder público y político y la lucha por la "dominación" del escenario de construcción comunicacional. El Estado y el sector privado están en una permanente lucha. Sin embargo, se priva al ciudadano de saber, en términos precisos qué debe validar y qué no: "[...] Hace necesario que los investigadores realicemos análisis cuidadosos de la remodelación de los espacios públicos y de los dispositivos que se pierden o recrean para el reconocimiento o la proscripción de las voces múltiples presentes en cada sociedad" (García Canclini, 1995).

Asimismo, Cristina Mata (2004) afirma que la globalización requiere una mirada de la comunicación desde el punto de vista del ejercicio de la ciudadanía, ya que este fenómeno afecta principalmente a las identidades y al multiculturalismo, tanto desde la perspectiva del consumo de bienes y servicios como desde el punto de vista de la comunicación. 
En este escenario, el ejercicio del poder se convierte en una situación de extrema importancia, porque las tensiones no se resumen a una lucha de clases, en la que se pueden identificar los extremos; de hecho, son un entretejido de representaciones que se autoconstruyen a medida que se van generando socialmente. No se puede pensar en la hegemonía como el instrumento de un determinado sector social, sino que se debe ver como un sistema de intercambio de poder que se genera en el propio ejercicio de los derechos civiles, y estos últimos sobrepasan ampliamente los de la mera convivencia entre pares: se desarrollan en función de las producciones culturales que son las que además validan los procesos.

Según Michel De Certeau (1996), "el Estado es una instancia de poder separada de la sociedad, constituida en un aparato jerárquico y burocrático, que enfrenta a la sociedad y la domina", esto es en parte la gran problemática de los estados modernos democráticos (de un marcado perfil populista). El enfrentamiento con el sector privado por el dominio de la información excluye a la sociedad de la discusión. Cabría preguntarse si los grandes grupos mediáticos funcionales al sistema capitalista de producción buscan la propagación de simbolismos ligados al consumo (tanto de objetos como de "cultura"), ¿qué procesos de simbolización busca el Estado?

Por otro lado, la sociedad necesita mecanismos de legitimación, que no encuentra ni en el sector publico (que le miente) ni en el sector privado (que lo seduce).

En este sentido, la categoría "clase social" se re-significa dentro de los nuevos contextos que habíamos mencionado (los que fundamentalmente están inmersos en el mundo de la globalización), dejando lugar a una nueva forma de sectorización (si se quiere) social, relacionada con las llamadas "minorías" y "mayorías", cuyo sistema de producción tiene que ver con las representaciones y no con los bienes exclusivamente materiales. Existe, por tanto, un "sistema de producción" que ya no está ligado a la posesión de los medios entendidos como capital, sino a los medios y modos de comunicación.

El fenómeno se define por la simpleza del mismo proceso: todos tienen acceso y todos tienen posibilidades de emitir mensajes mediatizados; hoy en día ya no se puede pensar en una restricción o un sometimiento de la capacidad de mediatizar mensajes, o de una mediación entre partes para poder hacerlos públicos: la misma presencia en la vía pública es entendida como un fenómeno de comunicación masivo, si el grupo supera cierta cantidad de personas.

Entonces, no se trata de las estratagemas, ni de los medios de comunicación, ni de los "modos de producción", sino de qué es lo que se considera hegemónico y qué se considera "sometido", dentro de los contextos representacionales y de intercambio simbólico y de cómo unas fuerzas hacen presión sobre las otras, con el fin de lograr legitimidad.

Williams (1981) afirma que "la hegemonía" no es el nivel superior articulado de la "ideología" ni tampoco sus formas de control consideradas habitualmente como 
"manipulación" o "adoctrinamiento". Con esto, la hegemonía puede dar cuenta de procesos más amplios pero a la vez más complejos, ya que no se circunscribe a la mera "dominación" de clase, definida en términos antiguos; de hecho, permite ampliar la discusión hacia todas las formas posibles de producción y reproducción cultural, tanto públicas como privadas.

Las palabras clave para comprender la construcción de mundo a través de los medios, teniendo en cuenta los procesos sociales y culturales y el papel de los poderes hegemónicos, son —como lo apunta Champagne (2000)— PRESENTACIÓN Y REPRESENTACIÓN.

En función de esto, y a modo de ejemplo puntual y específico, se pueden aislar las siguientes características que tienen las noticias construidas por los medios:

1. Anunciar el "acontecimiento" e inmediatamente su impacto.

2. La noticia es "explicada" a través de acontecimientos o hechos previos. Se buscan casi de inmediato las "causas" que desencadenaron los hechos. También puede suceder que no se considere "suficiente" o lo suficientemente influyente, por lo que se le "añaden" noticias o acontecimientos pasados (incluso de décadas atrás) que pueden ser comparables o complementarios. Este tipo de complementos sirve no solo para "ilustrar" la noticia, sino que se convierte en todo un universo de presentación y representación nuevo, que ayuda a reforzar las ideas principales.

3. Los periodistas y las redacciones no planifican las noticias, sino que evalúan el impacto a nivel social y "acuden en caliente" a los lugares de los hechos.

4. Las presentaciones y representaciones ponen acento sobre lo extraordinario.

5. Cuando se realiza periodismo de investigación, esta última no se realiza en el campo sino en la redacción, acudiendo al "lugar de los hechos" solo para ilustrar lo que se trazó con anterioridad.

6. Los mecanismos de participación ciudadana están mediados por la línea editorial del programa.

7. "La atención de los periodistas se concentra en los enfrentamientos más que en la situación objetiva que los provoca" (Champagne, 2000, p. 7).

El tema de la manipulación de la noticia televisiva ha sido motivo de debates ampliamente controversiales, sobre todo en el contexto de las profundas reestructuraciones que se vienen haciendo en lo referido a legislaciones y regulaciones de los medios en América Latina y Europa. Las posturas se pueden catalogar desde el punto de vista disciplinar que las genera, y por lo tanto no son cabales ni definitivas, pero encuentran una riqueza mayor desde la parcialidad, ya que permiten observar un mismo fenómeno desde múltiples puntos de vista.

Gran cantidad de investigadores y autores están "re-observando", tanto a nivel teórico como metodológico, los fenómenos mediáticos. Esto da pie a la necesaria 
revisión desde el punto de vista epistemológico de la problemática de los noticieros como agentes de generación de "opinión pública".

\section{Conclusiones}

La problemática de la investigación en temas de comunicación tiene varias aristas. Por un lado, se puede aislar la necesidad de hacer una revisión del material teórico "fundante", en segundo lugar, la revisión de una metodología acorde con los temas y las problemáticas y, por último, una mirada sobre la visión del futuro.

A modo de introducción se afirmaba que uno de los principales inconvenientes de la investigación en comunicación radica en la dificultad de conceptualizar a la comunicación en términos que no solo posibiliten una mirada crítica sobre los fenómenos que estudia, sino que posicionen a la disciplina en un campo que le permita tener una mirada holística del problema, permitiéndole diagnosticar situaciones desde el aporte de otras disciplinas y a su vez tener la capacidad de orientar las investigaciones en los caminos correctos, esto es, con la mirada puesta en el futuro, construyendo nuevas utopías.

La discusión -ya estéril en estos días - acerca de qué es la "realidad" y cómo el comunicador o investigador en comunicación influye o es influido por esta dificulta el avance y el derrocamiento de viejos y oxidados axiomas que ya han demostrado carecer de fundamentos.

Por otra parte, se suma a esta trágica situación otro tipo de discusión deudor de la sociología y de la antropología, que todavía tratan de explicar y comprender el fenómeno de la globalización y sus consecuencias. Se están aún reformulando las concepciones y las posturas, los espacios en los que se definen las luchas de clase y los terrenos en los que se dirime el ejercicio del poder, cuestiones que la comunicación requiere entender desesperadamente, para comprender a su vez sus propios problemas concretos.

El capitalismo ha trazado lineamientos que han convertido a los procesos comunicacionales "naturales" (también "necesarios") de un sistema social en productos de consumo. Desde esta nueva lógica, estamos obligados a cambiar la mirada urgentemente, enfocando nuestros esfuerzos en partir de la aceptación de esta realidad, para proponer nuevos escenarios acordes con ella, y no a querer "revertir" o contener un proceso que ya está en marcha.

La globalización se ha ocupado de traspasar las fronteras económicas y culturales, pero la investigación en relación con las ciencias sociales solo ha contemplado aspectos parciales generalmente ligados a los económicos o a lo cultural, sin observar los fenómenos de manera holística. A lo sumo se han creado categorías de análisis que apuntan a la feroz crítica a las hibridaciones culturales, como si ese fenómeno solo hubiera aparecido en la década de los noventa. 
No hay una forma literal de entendimiento y traducción de los mensajes y de su valoración y asignación de sentido: las producciones sociales/culturales son aisladas de las estrategias mediáticas, abriendo cada vez más la brecha entre los escenarios y los actores. Esto lleva a considerar las producciones culturales actuales como "híbridas", "deformes", "inadecuadas" e "importadas", ensanchando más la brecha entre los verdaderos indicadores de la problemática comunicacional, la comunidad de investigadores y los medios. Por un lado, entonces, se posiciona el ideal y el imaginario social-colectivo, con sus sistemas de representación y con su visión acerca de los productos culturales; por el otro están los medios, tratando de posicionar productos mediáticos en el mercado, y por último están los intelectuales, con sus viejos axiomas y sus viejas preguntas, ajenos a los ejes de importancia.

Por último, para concluir, es apropiada la siguiente cita de Jesús Martín Barbero:

Existe hoy un amplio consenso en las carreras de comunicación en torno a la necesidad de que la investigación supere el nivel de la generalidad denunciante para pasar a ser un ingrediente renovador de cómo y a quién hacer comunicación. Se trata de que la investigación ayude que la producción responda a demandas de comunicación que, aunque mediadas por la industria cultural, den expresión a necesidades colectivas y recojan matrices culturales de raigambre popular. Lo que exige transformar la docencia en un espacio de experimentación social posibilitando la apertura del trabajo académico a nuevos modos de relación con la 'vida real'. Pues lo que se torna irreal, especulativo y estéril en el trabajo académico no es la distancia indispensable que requiere la producción de conocimientos sino la irrelevancia social de los problemas que abordan y el formalismo de las soluciones tanto teóricas como prácticas. Entendemos entonces por experimentación social las estrategias de encuentro de la academia con la realidad comunicacional del país y la región. Los saberes que necesita el comunicador son tanto de su especializado oficio como los generales y públicos del intelectual.

\section{Bibliografía}

CASTORIADIS, Cornelius. Imaginario e imaginación en la encrucijada, En figuras de lo pensable.

CHAMPAGNE Patrick (2000). "La visión Mediática". En Bourdieu, Pierre. La miseria del Mundo. Bs. As. Fondo de Cultura Económica. http://www.nombrefalso.com.ar/index.php?pa

\footnotetext{
4 Martin Barbero, Jesús. "El oficio del Comunicador" en publicación Renglones, Número 48, abril-junio, 2001.
} 
DÍAZ LARRAÑAGA Nancy; PORTA Paula Inés. (2011) "Clases del 1 al 11: EPISTEMOLOGIA Y TRAYECTOS TEORICOS COMUNICACIONALES". UNQ. (Inédito)

FOLLARI, Roberto (2005) "La moldura en el espejo: encrucijadas epistemológicas de las ciencias de la comunicación". En Portal de la Comunicación. Espacio InCom-UAB, enero. http://www.portalcomunicacion.com/both/aab/txt/follari_2.pdf

FUENTES NAVARRO, Raúl. La investigación de la comunicación en América Latina: condiciones y perspectivas para el SXXI, en Diálogos, Felafacs, Lima.

GARCÍA CANCLINI, Néstor. El diálogo norte-sur en los estudios culturales, en Consumidores y Ciudadanos: conflictos multiculturales de la globalización. Grijalbo, México, 1995. Introducción.

HALL, Stuart (1994) "Estudios Culturales: dos paradigmas". En Revista Causas y azares N. ${ }^{\circ} \quad 1, \quad 1994 . \quad$ Traducción de Mirko Lauer. http://www.nombrefalso.com.ar/apuntes/pdf/hall_2.pdf

HUGHES, John y SHARROCK, Wes (1987, 1999), La filosofía de la investigación social. México: Fondo de Cultura Económica. Capítulo 3: "El positivismo y el lenguaje de la investigación social". Capítulo 5: "La alternativa interpretativista".

MARTÍN BARBERO, Jesús (1997) "Comunicación fin de siglo: ¿Para dónde va nuestra investigación?". En Telos N. ${ }^{\circ} \quad 47, \quad$ Madrid. http://www.comunicacionymedios.com/Reflexion/teorias/findesiglo.htm

MARTÍN BARBERO Jesús. "Comunicación el descentramiento de la modernidad". http://www.educar-argentina.com.ar/DIC2006/educ129.htm

MARX, K y Engels. Feuerbach: Primer Capitulo sobre la ideología alemana

MARX, K. Manuscritos económicos filosóficos de 1844.

MATA María Cristina. Comunicación, Ciudadanía y poder. Pistas para pensar su articulación. http://www.dialogosfelafacs.net/dialogos_epoca/pdf/64-05MariaMata.pdf

MATTELART, Armand y MATTELART, Michele. Historia de las Teorías de la Comunicación. 1995. Editorial Paidós, Barcelona, Edición en español 1997.

SCHUSTER, Félix G. Libro explicación y predicción: La validez del conocimiento en las ciencias sociales. Capítulo I. Las Ciencias Sociales. Aspectos Críticos. CLASCO. Ciudad Autónoma de Buenos Aires, Argentina, enero de 2005.

THOMPSON, John (1998) Los media y la modernidad. Barcelona: Paidós. Introducción y Cap. $\quad 1$ : Comunicación y contexto social. http://www.nombrefalso.com.ar/apunte.php?id=2

VASALLO DE LÓPEZ y FUENTES NAVARRO (2001) Comunicación, campo y objeto de estudio. Iteso, México.

VIZER, E. (2003) La trama (in)visible de la vida social. Comunicación, sentido y realidad. La Crujía. Bs. As. Introd. y Cap. I.

WALLERSTEIN, Immanuel. Conocer el mundo, saber el mundo: el fin de lo aprendido. Una ciencia social para el siglo XXI. Siglo XXI editores. 
De Prácticas y discursos/ Universidad Nacional del Nordeste/ Centro de Estudios Sociales

(1). WILLIAMS, Raymond (1980) Teoría cultural, en Marxismo y Literatura. Barcelona. Península.

WOLTON, Dominique (1999) "La comunicación en el centro de la modernidad. Un debate teórico fundamental". En Internet, ¿y después?: una teoría crítica de los nuevos medios de comunicación. Barcelona: Gedisa. pp. 35-67. 